\title{
Accurate and Process-Tolerant Resistive Load
}

\author{
Batuhan Sutbas $^{(}$, Ekmel Ozbay, Member, IEEE, and Abdullah Atalar, Fellow, IEEE
}

\begin{abstract}
Resistive terminations cannot preserve high-quality matching at high frequencies due to the parasitic effects of the nonideal resistor. Moreover, resistance values of the termination resistors in integrated circuits are subject to process variations. Therefore, it is difficult to obtain accurate and process-tolerant terminations that are crucial for high performance in microwave circuits. We propose a new resistive network that compensates for the high-frequency parasitic effects of the resistors to improve the bandwidth of the termination. In addition to maintaining accuracy, the presented network provides tolerance to variation in the resistor values. The accuracy and tolerance of the proposed structure is analytically shown and experimentally verified by three test structures at the $\mathrm{X}$-band fabricated on a GaN technology. The experimental results show that a small size and wideband $50-\Omega$ load with a return loss better than $25 \mathrm{~dB}$ can be obtained, while the resistor value changes $\pm 30 \%$.
\end{abstract}

Index Terms-Accurate, integrated circuit, parasitic effect, process-temperature variation, resistor, sheet resistance, termination, tolerance, via inductance, wideband.

\section{INTRODUCTION}

$\mathbf{R}$ ESISTORS are used in the load termination networks of microwave circuits, such as couplers, dividers, and balanced and distributed amplifiers. Bandwidth and performance of the circuits are significantly dependent on the load accuracy. However, parasitic effects associated with the resistor and changes in the resistor value due to process variations limit the accuracy of the load.

A real resistor exhibits parasitic capacitive or inductive effects limiting the useful bandwidth. Early efforts focused on avoiding a direct ground connection to reduce inductive effects and achieve broadband characteristics [1]. Researchers used nongrounded loads with open [2] and radial [3] stubs along with branched [4], compensated [5], and ellipse-shaped [6] terminations. With known resistor characteristics at high frequencies, it is also possible to design an additional circuitry

Manuscript received November 24, 2019; revised January 19, 2020; accepted March 3, 2020. Date of publication April 27, 2020; date of current version July 1, 2020. (Corresponding author: Batuhan Sutbas.)

Batuhan Sutbas was with the Department of Electrical and Electronics Engineering, Bilkent University, 06800 Ankara, Turkey, and also with the Nanotechnology Research Center, Bilkent University, 06800 Ankara, Turkey. $\mathrm{He}$ is now with the Circuit Design Department, Innovations for High Performance Microelectronics, 15236 Frankfurt (Oder), Germany (e-mail: sutbas@ee.bilkent.edu.tr).

Ekmel Ozbay is with the Nanotechnology Research Center, Bilkent University, 06800 Ankara, Turkey, and also with the Department of Electrical and Electronics Engineering, Bilkent University, 06800 Ankara, Turkey (e-mail: ekmel@ee.bilkent.edu.tr).

Abdullah Atalar is with the Department of Electrical and Electronics Engineering, Bilkent University, 06800 Ankara, Turkey (e-mail: atalar@ee.bilkent.edu.tr).

Color versions of one or more of the figures in this article are available online at http://ieeexplore.ieee.org.

Digital Object Identifier 10.1109/TMTT.2020.2986207 that cancels the parasitic effects and increases the highest operating frequency [7]-[9]. However, these approaches require an accurate model for the parasitics of the resistive elements and they do not compensate for the manufacturing variations in the value of the intrinsic resistor, limiting their usefulness. More recently, the emerging use of carbon nanotubes [10] and 2-D inkjet printing [11] have also facilitated unconventional methods to fabricate wideband loads.

The resistance value of the intrinsic resistor is somewhat variable since the fabrication processes have tolerance and repeatability limits. For instance, the thickness of the thin-film resistors may be different in successive processes or even in wafers from the same fabrication batch which changes the sheet resistance value. For higher yield and better circuit performance, this variation has to be considered at the design step and the tolerance of the load network needs to be increased. This procedure is vital, especially in the case of integrated circuits, as it is difficult to have a tuning mechanism after the fabrication.

Resistance variances of discrete resistors in a discrete circuit are independent of each other. On the contrary, the resistances of resistors in an integrated circuit or in a thick-film circuit are related to each other by the fabrication process itself and change in the same direction. Resistors in close proximity have almost identical sheet resistance regardless of the deviation from the nominal value. When the process has a higher sheet resistance than its nominal value, all resistors in the same integrated circuit have higher resistance values. Similarly, high-frequency parasitic capacitors of the resistors are dependent on the substrate that they share, hence, resistors in the same area show a similar parasitic capacitance. Also, when the resistors are identical in size, they exhibit an equal parasitic inductance.

In this article, we use this correlated behavior of resistors in integrated and thick-film circuits to realize a novel accurate resistive load with parasitic effect cancellation, which does not require previous knowledge of the resistor parasitics. Although our proposed technique is not as useful for providing tolerance to variations in discrete circuits, it can still achieve parasitic effect cancellation and can be utilized in discrete load networks. The presented load network can easily be integrated with RF components, such as couplers and balanced amplifiers for performance, bandwidth, and tolerance improvement.

Our proposed structure consists of two resistors in parallel where one of the resistors is transformed by a quarter-wave length transmission line. The idea is that the change in the resistances of the two parallel resistors is in the opposite direction after quarter-wave transformation, providing tolerance to variations in resistor values in a wide bandwidth. 


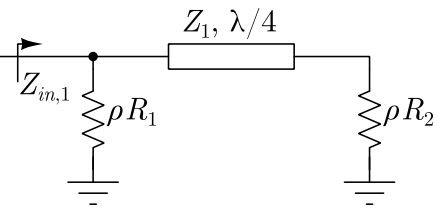

(a)

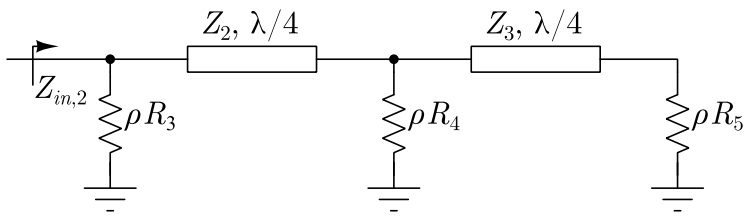

(b)

Fig. 1. Schematic of the proposed accurate and process tolerant resistive load network. (a) First-order $(N=1)$. (b) Second-order $(N=2)$.

Moreover, our proposed topology compensates for the parasitic effects of the resistor including via inductance, improving the high-frequency response and enabling higher bandwidth. As the integrated circuit frequency moves toward the millimeter-wave region, it becomes a necessity to use such a structure since parasitic components dominate the intrinsic resistor. One drawback of the presented approach is that the circuit occupies more area because it requires a quarter-wave length transmission line. Nevertheless, the additional high-impedance transmission line can be meandered for a smaller size without bandwidth degradation. We demonstrate that the miniaturized structure also achieves high performance in a wideband with high sheet resistance tolerance.

The relation between sheet resistance tolerance, return loss, and bandwidth is shown analytically. We derive an equation for the improvement in return loss compared with a single resistor when the resistor model with parasitic effects is incorporated. Three test structures are fabricated with a $\mathrm{GaN}$ on $\mathrm{SiC}$ microstrip monolithic microwave integrated circuit (MMIC) technology and demonstrate that our proposed technique is applicable at the X-band. Studies have shown that the reliability and accuracy of sheet resistance in a fabrication process can be improved to implement accurate loads [12], [13]. However, to the best of our knowledge, this is the first work that obtains a sheet resistance tolerance using an improved design.

\section{ANALYSIS AND DESIGN}

\section{A. Analysis for Sheet Resistance Variance}

Our proposed network for the resistive termination is shown in Fig. 1(a). $R_{1}$ and $R_{2}$ are two shunt resistors without parasitics separated by a quarter-wavelength transmission line with a characteristic impedance of $Z_{1}$. We should have

$$
Z_{1}=R_{1}=R_{2}=2 Z_{0}
$$

with perfect matching. In our analysis, $\rho$ represents the normalized sheet resistance that is ideally equal to 1 but is defined by the manufacturing process. For $\rho<1$, both $\rho R_{1}$ and $\rho R_{2}$ have a resistance value below the nominal design resistance values. However, the impedance seen into the network, $Z_{\text {in, } 1}$, does not drop by the same ratio because the quarter-wave transformed impedance $Z_{1}{ }^{2} /\left(\rho R_{2}\right)$ increases and compensates for the decrease in $\rho R_{1}$. Similarly, for $\rho>1$, the deviations from nominal values are in opposite direction and the overall impedance changes by a much smaller amount. The presence of a quarter-wavelength line does not cause a bandwidth reduction since the load and the characteristic impedance have nearly the same values. The proposed structure uses the fact that the variation in $Z_{1}$ is considerably less than that in $\rho$.

Sheet resistance tolerance and bandwidth of the circuit can be improved further by adding another shunt resistor and quarter-wavelength transmission line, as shown in Fig. 1(b). Here, $R_{4}, Z_{3}$, and $R_{5}$ form a network similar to the first-order load. The additional quarter-wavelength line $Z_{2}$ transforms the impedance seen into this network to compensate for the change in $\rho R_{3}$, greatly reducing the overall deviation in $Z_{\text {in, } 2}$.

To obtain a first-order load network with reflection coefficient smaller than a limit level of $\delta$, the following inequality has to hold:

$$
\left|\Gamma_{\text {in, } 1}\right|=\left|\frac{Z_{\text {in, } 1}-Z_{0}}{Z_{\text {in }, 1}+Z_{0}}\right|<\delta
$$

where the impedance seen into the network for (1) is

$$
Z_{\text {in }, 1}=2 Z_{0} \frac{\rho^{2}+j T \rho}{2 \rho+j T\left(1+\rho^{2}\right)} .
$$

Here, $Z_{0}$ is the reference characteristic impedance and $T=$ $\tan (\pi \hat{f} / 2)$ with $\hat{f}=f / f_{0}$, where $f_{0}$ is the design center frequency. Then, the reflection coefficient simplifies to

$$
\left|\Gamma_{\text {in }, 1}\right|=\sqrt{\frac{(\rho-1)^{2}}{(\rho+1)^{2}} \frac{4 \rho^{2}+T^{2}(\rho-1)^{2}}{4 \rho^{2}+T^{2}(\rho+1)^{2}}}<\delta
$$

and the fractional bandwidth $\left(\mathrm{FBW}=\mathrm{BW} / f_{0}\right)$ in terms of $\rho$ and $\delta$ becomes

$$
\mathrm{FBW}=\left|2-\frac{4}{\pi} \arctan \sqrt{4 \rho^{2} \frac{(\rho-1)^{2}-\delta^{2}(\rho+1)^{2}}{\delta^{2}(\rho+1)^{4}-(\rho-1)^{4}}}\right| .
$$

Although a single ideal termination resistor with resistance $Z_{0}$ does not have bandwidth limitation, for $\rho \neq 1$, it can only achieve a reflection coefficient of $\Gamma_{0}$ given by

$$
\left|\Gamma_{0}\right|=\frac{\left|\rho Z_{0}-Z_{0}\right|}{\rho Z_{0}+Z_{0}}=\frac{|\rho-1|}{\rho+1}<\delta .
$$

For a desired matching level of $\delta=0.056$ (25 $\mathrm{dB}$ return loss), the sheet resistance tolerance should be less than $12 \%$.

The resistor and the line impedance values given in (1) can be optimized to achieve a wider bandwidth, by sacrificing the perfect match condition at the center frequency. In the general case, the impedance seen into the first-order network is

$$
Z_{\text {in }, 1}=\frac{Z_{1} \rho^{2} R_{1} R_{2}+j T Z_{1}^{2} \rho R_{1}}{Z_{1} \rho\left(R_{1}+R_{2}\right)+j T\left(Z_{1}^{2}+\rho^{2} R_{1} R_{2}\right)} .
$$

For instance, a first-order load of Fig. 1(a) with $Z_{1}=$ $1.9 Z_{0}, R_{1}=2.4 Z_{0}$, and $R_{2}=1.8 Z_{0}$ achieves a $25-\mathrm{dB}$ return loss in a $30 \%$ FBW despite a $48 \%$ variation in sheet resistance, while a circuit using (1) could tolerate up to $34 \%$ variation within the same bandwidth.

In Fig. 2, we plot the 25-dB FBW of the first-order network based on (5) along with optimized ${ }^{1}$ first- and second-order

\footnotetext{
${ }^{1}$ ADS 2017.01, Keysight Technologies, Inc., Santa Rosa, CA, USA.
} 


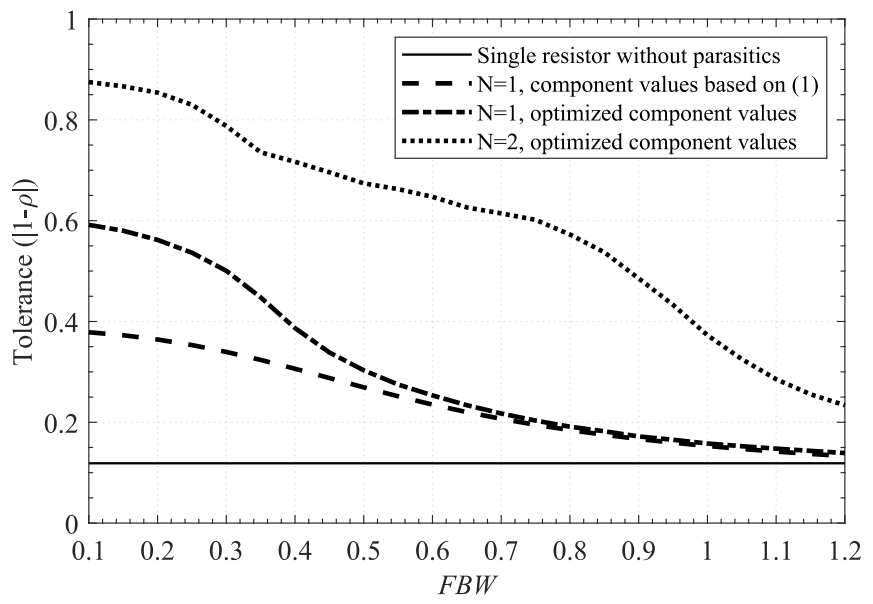

Fig. 2. Achievable sheet resistance tolerance level versus FBW for 25-dB $(\delta=0.056)$ return loss using a single resistor without parasitics and using our proposed network.

networks. The proposed networks always provide better sheet resistance tolerance levels than a single resistor, but the improvement gradually decreases as the bandwidth of operation is increased. For narrowband applications, the proposed first-order network can provide sheet resistance tolerances higher than $60 \%$ for a $25-\mathrm{dB}$ load compared with $12 \%$ of a single resistor without parasitics.

It is possible to add more sections and increase the order of the network $N$ for further improvement in tolerance and matching levels. However, we note that the improvement diminishes and becomes insignificant after $N=3$. The additional degrees of freedom in higher order networks can be used to find the component values that are more suitable or easily realizable. For example, a second-order load of Fig. 1(b) with $Z_{2}=1.3 Z_{0}, Z_{3}=2.5 Z_{0}, R_{3}=4.6 Z_{0}, R_{4}=2.8 Z_{0}$, and $R_{5}=2 Z_{0}$ achieves a $25-\mathrm{dB}$ return loss in an FBW of $80 \%$ despite more than $50 \%$ variation in sheet resistance.

The very high sheet resistance tolerance obtained by the network not only increases the accuracy but also enables the proposed circuits to be placed inside the normally unusable area in wafers with high variation in process parameters, essentially increasing the number of devices fabricated per wafer. Furthermore, the high tolerance enables the load circuit to work with high temperature variations.

In addition to the increase in sheet resistance tolerance, the proposed network has an increased power-handling capability due to the higher number of resistors connected to the ground.

\section{B. Analysis for Parasitic Effect Cancellation}

The lumped element model of a shunt resistor is shown in Fig. 3: $R_{i}$ represents the intrinsic resistance, $L_{i}$ is the intrinsic inductance to account for the finite electrical length of the resistor, $C_{g}$ is the capacitance, and $L_{v}$ is the via inductance.

We replace $R_{1}$ and $R_{2}$ in our first-order network with a resistor model with unknown parameters to analyze the parasitic effect cancellation, assuming that the real part of the impedance seen looking into the resistor is only dependent

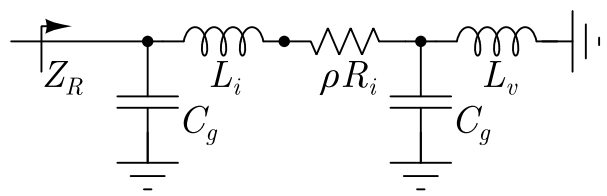

Fig. 3. Lumped element model of a shunt thin-film resistor.

on the intrinsic resistance, $\operatorname{Re}\left\{Z_{R}\right\}=\rho R_{i}$. The reflection coefficient of a single resistor with respect to $Z_{0}$ becomes

$$
\left|\Gamma_{1}\right|=\left|\frac{\bar{Z}-1}{\bar{Z}+1}\right|
$$

where $\bar{Z}=\rho+j \bar{X}$ is the normalized impedance and $\bar{X}=\operatorname{Im}\left\{Z_{R} / Z_{0}\right\}$ is the normalized parasitic reactance. For a comparison with our proposed structure, we use two of such resistors with an intrinsic resistance of $2 \rho Z_{0}$, both of which have a normalized parasitic reactance of $2 \bar{X}$, separated by a quarter-wavelength transmission line with $Z_{1}=2 \alpha Z_{0}$ as in (1). Here, we further consider a variation in the characteristic impedance of the transmission line by a factor of $\alpha$. We find the reflection coefficient with respect to $Z_{0}$ as

$$
\left|\Gamma_{2}\right|=\left|\frac{\overline{Z_{T}} / / 2 \bar{Z}-1}{\overline{Z_{T}} / / 2 \bar{Z}+1}\right|
$$

where the normalized quarter-wave transformed impedance is

$$
\overline{Z_{T}}=2 \alpha \frac{\bar{Z}+j \alpha T}{\alpha-j T \bar{Z}} .
$$

Then, the reflection coefficient in terms of $\alpha, \bar{Z}$, and $T$ is

$$
\left|\Gamma_{2}\right|=\left|\frac{\alpha^{2} T(2 \bar{Z}-1)-T \bar{Z}^{2}-j 2 \alpha\left(\bar{Z}^{2}-\bar{Z}\right)}{\alpha^{2} T(2 \bar{Z}+1)+T \bar{Z}^{2}-j 2 \alpha\left(\bar{Z}^{2}+\bar{Z}\right)}\right| .
$$

The characteristic impedance of a line is a more stable parameter since it changes more slowly with deviations in the physical parameters, such as the height and dielectric constant of the substrate and the width of the transmission line. Hence, the sheet resistance variance and parasitic effects are more dominant. The reflection coefficients for $0.95 \leq \alpha \leq 1.05$ are calculated using the resistor model parameters for our substrate and plotted in Fig. 4 for a center frequency of $10 \mathrm{GHz}$ along with the reflection coefficient of a single resistor.

The proposed network achieves better matching than a single resistor at all frequencies even in the case of an unlikely deviation from the desired line impedance. With $\alpha=1$, the improvement in return loss can be simplified as

$$
\left|\frac{\Gamma_{2}}{\Gamma_{1}}\right|=\left|\frac{T(\bar{Z}-1)+j 2 \bar{Z}}{T(\bar{Z}+1)+j 2 \bar{Z}}\right|
$$

and is $0 \mathrm{~dB}$ at the worst case (for $T=0$ ), which shows that our proposed network never performs worse than a single resistor

$$
\lim _{T \rightarrow \infty}\left|\frac{\Gamma_{2}}{\Gamma_{1}}\right|=\left|\frac{\bar{Z}-1}{\bar{Z}+1}\right|=\left|\Gamma_{1}\right| \text {. }
$$

For odd multiples of the center frequency (when $T \rightarrow \infty$ ), (13) shows that the proposed structure doubles the return loss 


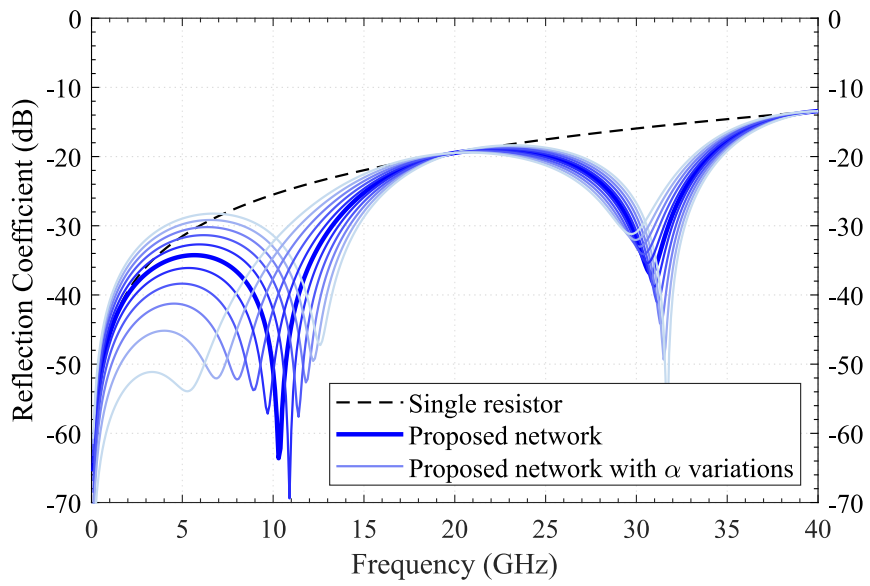

Fig. 4. Reflection coefficients of a single resistor and the proposed network $(N=1)$ with variations in the transmission-line characteristic impedance as a function of frequency using the extracted model parameters $\left(L_{i}=77 \mathrm{pH}\right.$, $C_{g}=11 \mathrm{fF}$, and $L_{v}=35 \mathrm{pH}$ ) of a $53-\mu \mathrm{m}$-wide high power $50-\Omega$ resistor.

(in dB-scale) of a single resistor, irrespective of the sheet resistance variance and the parasitic values.

The proposed method can be combined with other parasitic cancellation techniques given in the literature to achieve even higher performance levels with an additional benefit of sheet resistance tolerance and higher power-handling capability. For instance, $N+1$ of the matched loads with radial stubs described in [3] can be separated by $N$ quarter-wavelength lines in the form of an $N$ order accurate and process-tolerant resistive load network to obtain superior performance than the original matched loads with the additional benefit of having tolerance to variation in process and operating conditions. We note that the bandwidth of the original parasitic cancellation technique will not be reduced because our analysis treats the single resistor as a black box, and thus, (12) and its implications apply to any type of termination network. On a separate note, the parasitic effect cancellation of the presented technique can be utilized in discrete circuits by selecting the resistor parts from the same supplier, as they show a similar parasitic behavior.

\section{EXPERIMENTAL RESULTS}

Three test structures (TS-A, TS-B, and TS-C) at $10 \mathrm{GHz}$ are fabricated to demonstrate the proposed technique that provides sheet resistance tolerance and parasitic effect cancellation. Each test structure is composed of an $N=1$ termination network with three variants. The first variant uses the nominal resistor, whereas the other two variants have deliberately changed resistor values to simulate the manufacturing variance.

In TS-A, shown in Fig. 5(a), the nominal resistor value is $R_{a}=105 \Omega$. The quarter-wave length line widths are $10 \mu \mathrm{m}$ $\left(Z_{a}=102 \Omega\right)$. SEM image of $R_{a}$ is shown in Fig. 6 with the measured length and width of 35 and $10 \mu \mathrm{m}$, respectively.

In TS-B, shown in Fig. 5(b), we have $R_{b}=100 \Omega$. The quarter-wave length line widths are $12 \mu \mathrm{m}\left(Z_{b}=99 \Omega\right)$.

The compact loads in test structure TS-C, shown in Fig. 7, use meandered lines for smaller footprints compared with the

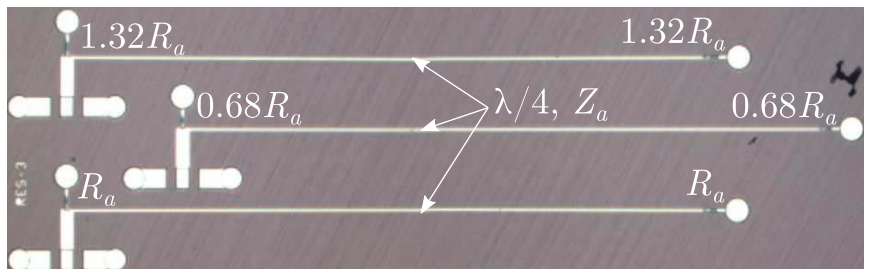

(a)

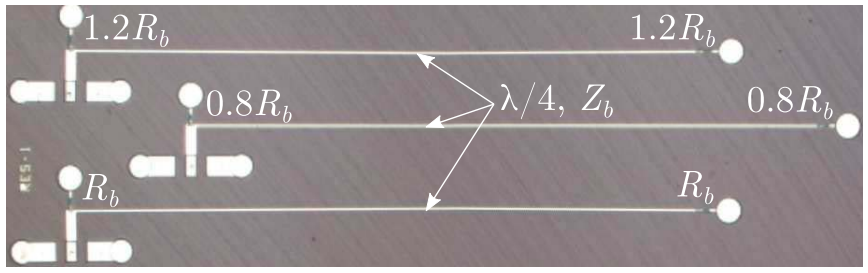

(b)

Fig. 5. Microscope photograph of (a) TS-A with $\pm 32 \%$ resistance variation and (b) TS-B with $\pm 20 \%$ resistance variation.

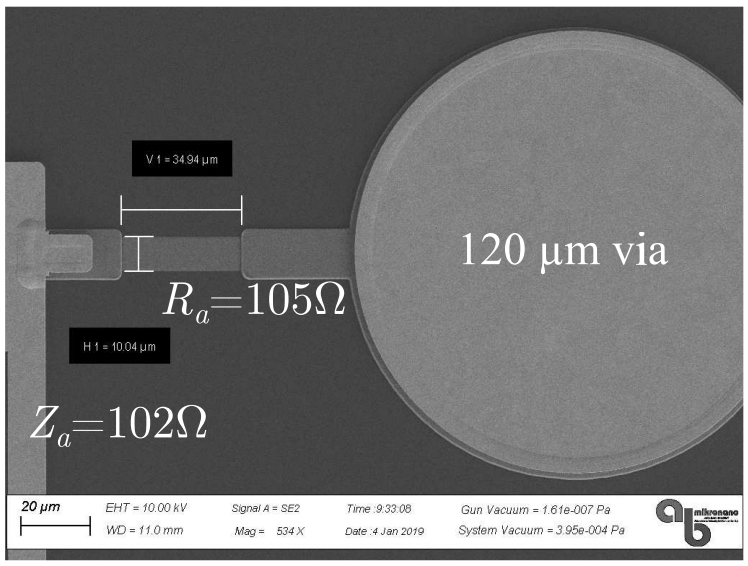

Fig. 6. SEM image of the nominal resistor in the first variant of TS-A.

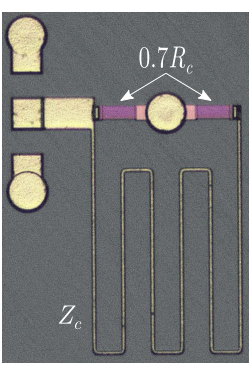

(a)

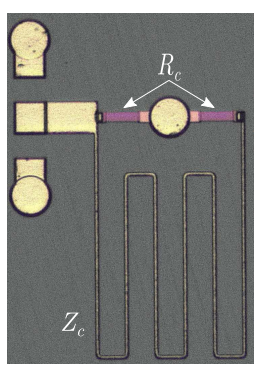

(b)

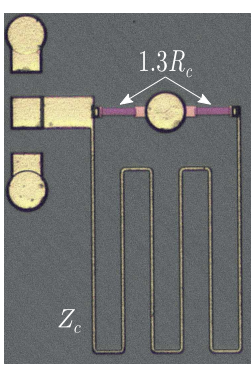

(c)
Fig. 7. Microscope photograph of TS-C with (a) smaller resistances $(\rho=0.7)$, (b) nominal resistances $(\rho=1)$, and (c) larger resistances $(\rho=1.3)$.

loads in TS-A and TS-B. The resistors are oriented in the same direction next to each other and connected to the ground using the same via hole. We use $R_{c}=101 \Omega$ and $Z_{c}=102 \Omega$. The sizes of the termination networks in TS-C without the measurement pads are less than $0.47 \mathrm{~mm} \times 0.82 \mathrm{~mm}$.

All the test structures with their variations are fabricated using our in-house GaN monolithic microwave integrated circuit (MMIC) process with two metal (Au) layers and a 
TABLE I

MEASURED BANDWIDTH FOR 25-dB RETURN LOSS

\begin{tabular}{|c|c|c|c|c|c|c|c|c|c|}
\hline Type & \multicolumn{3}{|c|}{ TS-A } & \multicolumn{3}{c|}{ TS-B } & \multicolumn{3}{c|}{ TS-C } \\
\hline $\boldsymbol{\rho}$ & 0.68 & 1 & 1.32 & 0.8 & 1 & 1.2 & 0.7 & 1 & 1.3 \\
\hline $\boldsymbol{f}_{\boldsymbol{L}}(\mathbf{G H z})$ & 8.1 & $\mathrm{dc}$ & 8.7 & 5.7 & $\mathrm{dc}$ & 6.9 & 8.2 & $\mathrm{dc}$ & 9.0 \\
\hline $\boldsymbol{f}_{\boldsymbol{H}}(\mathbf{G H z})$ & 12.3 & $25+$ & 12.4 & 12.8 & $25+$ & 13.4 & 11.1 & 34.4 & 11.4 \\
\hline $\boldsymbol{F B} \boldsymbol{B}$ & 0.41 & 1 & 0.35 & 0.77 & 1 & 0.64 & 0.30 & 1 & 0.24 \\
\hline
\end{tabular}

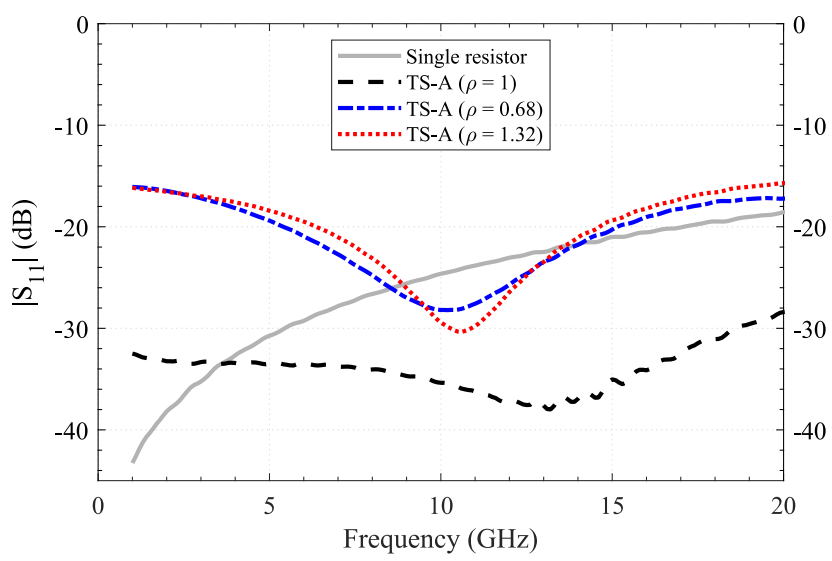

Fig. 8. Measured reflection coefficients of TS-A as a function of frequency.

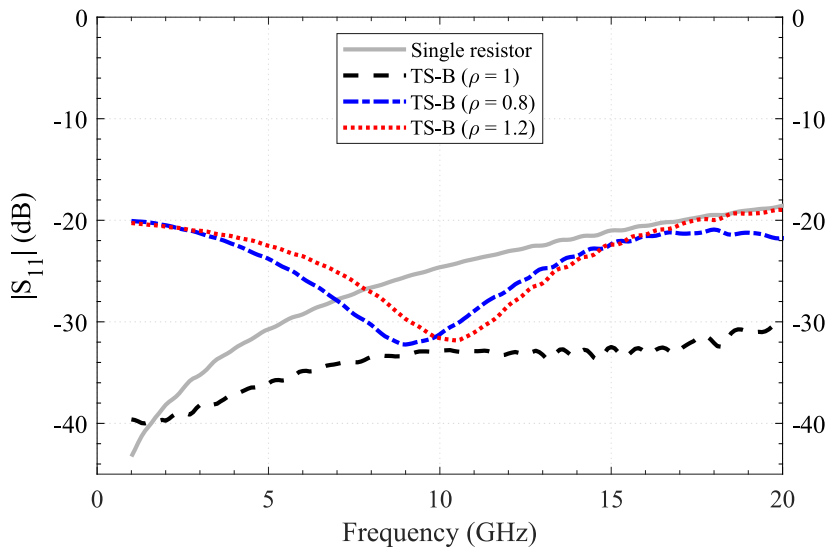

Fig. 9. Measured reflection coefficients of TS-B as a function of frequency.

backside via-hole process. $\mathrm{SiC}$ substrate is $100-\mu \mathrm{m}$-thick with a dielectric constant of 9.7. Thin-film resistors are formed using TaN sputtering with a thickness of $90 \mathrm{~nm}$. The nominal sheet resistance of the process technology is $30 \Omega / \square$. Quarterwave length at $10 \mathrm{GHz}$ is approximately $3.3 \mathrm{~mm}$.

Calibration and measurements are performed using Cascade $^{2}$ RF wafer probe station, GGB ${ }^{3}$ GSG150 picoprobes, and $R \& S^{4}$ ZVA40 vector network analyzer. A summary of the experimental results is presented in Table I.

Fig. 8 shows the measured S-parameters of TS-A with different resistor values achieving return losses better than $25 \mathrm{~dB}$ from 8.7 to $12.3 \mathrm{GHz}$ (an FBW of $34 \%$ ) with a sheet resistance tolerance of $32 \%$. Fig. 9 shows that TS-B achieves

\footnotetext{
${ }^{2}$ Cascade Microtech, Inc., Beaverton, OR, USA.

${ }^{3}$ GGB Industries, Inc., Naples, FL, USA.

${ }^{4}$ Rohde \& Schwarz GmbH \& Company KG, Munich, Germany.
}

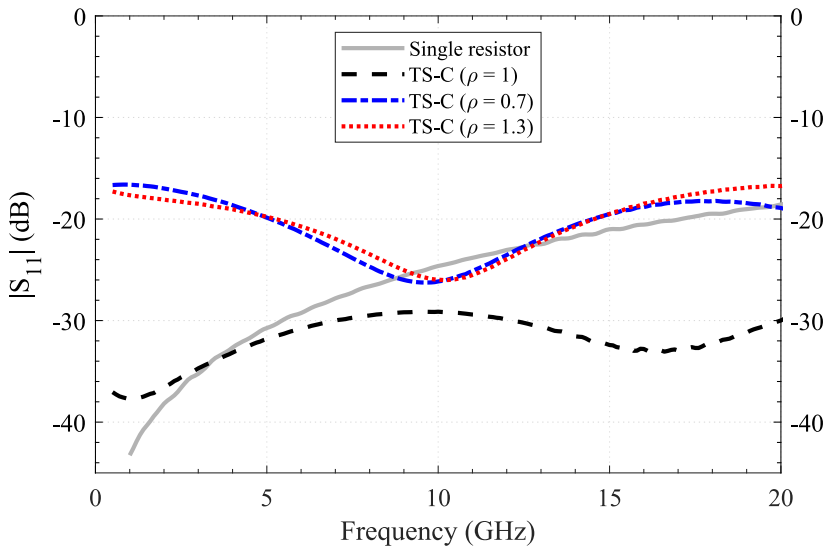

Fig. 10. Measured reflection coefficients of TS-C as a function of frequency.

the return losses better than $25 \mathrm{~dB}$ from 6.9 to $12.8 \mathrm{GHz}$ (an FBW of 60\%) with a sheet resistance tolerance of $20 \%$. Fig. 10 shows the measured reflection coefficients of TS-C, demonstrating $20-\mathrm{dB}$ matching from 5.5 to $14.6 \mathrm{GHz}$ (an FBW of $91 \%$ ), even though the resistor values are changed by $\pm 30 \%$.

For comparison, the measured reflection coefficient of a single nominal $50-\Omega$ resistor placed next to the test structures is also provided on each figure. Due to its high-frequency parasitic effects, the single resistor cannot present a $25-\mathrm{dB}$ load at frequencies higher than $9.6 \mathrm{GHz}$. All proposed test structures with nominal resistors achieve almost $30-\mathrm{dB}$ return loss from dc to $20 \mathrm{GHz}$.

\section{CONCLUSION}

A novel resistive load network, which provides high accuracy and sheet resistance tolerance, is introduced. The presented technique is useful for adding process tolerance to balanced and distributed amplifiers, as well as couplers and dividers while improving their high-frequency performance. Parasitic effect cancellation of the method is independent of the fabrication technology and the underlying resistor model, which makes the load circuit suitable for high-frequency RF components in both discrete and integrated circuits.

\section{REFERENCES}

[1] L. J. P. Linner and H. B. Lunden, "Theory and design of broad-band nongrounded matched loads for planar circuits," IEEE Trans. Microw. Theory Techn., vol. MTT-34, no. 8, pp. 892-896, Aug. 1986.

[2] L. J. P. Linner, H. B. Lunden, and M. A. Larsson, "Design of MIC broadband loads and attenuators," in Proc. 14th Eur. Microw. Conf., Liege, Belgium, Sep. 1984, pp. 503-509.

[3] F. Giannini, C. Paoloni, and M. Ruggieri, "A very broadband matched termination utilizing non-grounded radial lines," in Proc. 17th Eur Microw. Conf., Rome, Italy, Oct. 1987, pp. 1027-1031.

[4] H. Ashoka and A. M. Khilla, "New type of broadband termination," in Proc. 18th Eur. Microw. Conf., Stockholm, Sweden, Oct. 1988, pp. 583-587.

[5] C. Buoli, "Microstrip attenuators and terminations realized with periodic electromagnetic structures," in Proc. 18th Eur. Microw. Conf., Stockholm, Sweden, Oct. 1988, pp. 593-598.

[6] R. R. Monje, V. Vassilev, A. Pavolotsky, and V. Belitsky, "High quality microstrip termination for MMIC and millimeter-wave applications," in IEEE MTT-S Int. Microw. Symp. Dig., Long Beach, CA, USA, Jun. 2005, pp. $1827-1830$ 
[7] N. Jain and D. Wells, "Design of a DC-to-90-GHz resistive load," IEEE Microw. Guided Wave Lett., vol. 9, no. 2, pp. 69-70, Feb. 1999.

[8] B. Lopez-Berrocal, J. de Oliva-Rubio, and I. Molina-Fernandez, "Design and implementation of $\mathrm{DC}-20-\mathrm{GHz}$ lumped resistor matched loads for planar microwave circuits," IEEE Trans. Microw. Theory Techn., vol. 57, no. 10 , pp. 2439-2443, Oct. 2009.

[9] A.-V.-H. Pham et al., "Development of integral passive components for multilayer organic MCMs at millimeter wave frequencies," IEEE Trans. Adv. Packag., vol. 25, no. 1, pp. 98-101, Feb. 2002.

[10] J.-K. Chuang, R.-Y. Fang, Y.-J. Huang, Y.-C. Lee, C.-L. Wang, and K.-Y. Lee, "A broadband-matched load using multiwalled carbon nanotubes," IEEE Trans. Nanotechnol., vol. 12, no. 6, pp. 1213-1218, Nov. 2013.

[11] Y. Morimoto, M. Memarian, X. Li, and T. Itoh, "Open-end microstrip line terminations using lossy gray-scale inkjet printing," IEEE Trans. Microw. Theory Techn., vol. 65, no. 12, pp. 4861-4870, Dec. 2017.

[12] S. Vinayak, H. P. Vyas, K. Muraleedharan, and V. D. Vankar, "Ni-Cr thin film resistor fabrication for GaAs monolithic microwave integrated circuits," Thin Solid Films, vol. 514, nos. 1-2, pp. 52-57, Aug. 2006.

[13] J. Balcells-Ventura, T. Klein, P. Uhlig, C. Gunner, and R. Kulke, "Tolerance-optimized RF structures in LTCC for $\mathrm{mm}$-wave frequencies applications," J. Ceram. Sci. Tech., vol. 6, no. 4, pp. 267-272, Apr. 2015.

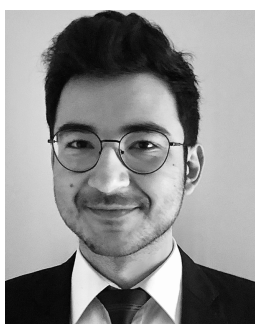

Batuhan Sutbas received the B.S. and M.S. degrees in electrical and electronics engineering from Bilkent University, Ankara, Turkey, in 2016 and 2019, respectively. $\mathrm{He}$ is currently pursuing the Ph.D. degree at the Innovations for High Performance Microelectronics (IHP), Frankfurt (Oder), Germany.

From 2016 to 2019, he was a Design Engineer with the Nanotechnology Research Center (NANOTAM), Bilkent University, Ankara, and AB-MicroNano Inc., Ankara, where he was involved in the design and development of GaN-based high-power amplifier monolithic microwave integrated circuits. Since 2019, he has been a Scientist with IHP. His current research interests include the design of low-power millimeter-wave components and radar transceivers in SiGe BiCMOS technology.

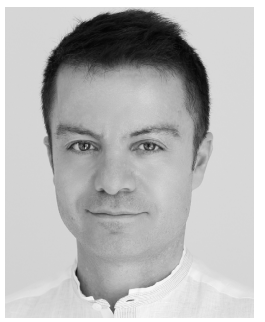

Ekmel Ozbay (Member, IEEE) received the M.S. and Ph.D. degrees in electrical engineering from Stanford University, Stanford, CA, USA, in 1989 and 1992, respectively.

He held a post-doctoral position at Stanford University. He worked as a Scientist with Iowa State University, Ames, IA, USA. He joined Bilkent University, Ankara, Turkey, in 1995, where he is currently a Full Professor with the Department of Physics and the Department of Electrical and Electronics Engineering. In 2003, he founded the Nanotechnology Research Center (NANOTAM), Bilkent University, where he leads a research group working on nanophotonics, nanometamaterials, nanoelectronics, and GaN-based devices. He is also the CEO of a spin-off company: AB-MicroNano Inc., which is founded to commercialize the technologies developed at NANOTAM. He has published more than 505 articles in SCI journals. His articles have received more than 17000 SCI citations with an $\mathrm{H}$-index of 59.

Dr. Ozbay was a recipient of the Adolph Lomb Medal of OSA in 1997 and the European Union Descartes Science Award in 2005. He has given more than 165 invited talks in international conferences. He served as an Editor for Optics Letters, PNFA, SPIE JNP, and the IEEE JOURNAL OF QUANTUM ELECTRONICS.

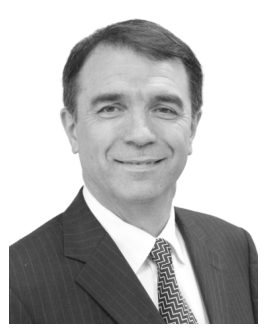

Abdullah Atalar (Fellow, IEEE) received the B.S. degree from Middle East Technical University, Ankara, Turkey, in 1974, and the M.S. and Ph.D. degrees from Stanford University, Stanford, CA USA, in 1976 and 1978, respectively, all in electrical engineering.

From 1978 to 1980, he was first a Post-Doctoral Fellow and later an Engineering Research Associate with Stanford University. For about one year, he worked at Hewlett Packard Labs, Palo Alto, CA, USA. From 1980 to 1986 , he was on the faculty of the Middle East Technical University as an Assistant Professor. In 1983 , on leave from the University, he worked for Ernst Leitz Wetzlar (now Leica), Wetzlar, Germany. In 1986, he joined the Department of Electrical and Electronics Engineering, Bilkent University, Ankara, as the Chairman. He served in the founding of the Department of Electrical and Electronics Engineering, where he is currently a Professor. In 1995, he was a Visiting Professor with Stanford University. From 1996 to 2010, he was the Provost with Bilkent University, where he is also the Rector. His current research interests include microwave electronics and micromachined sensors.

Dr. Atalar has been a member of the Turkish Academy of Sciences since 1997. He served as a member of the Science Board of the Turkish Scientific Research Council (TUBITAK) from 2004 to 2011. He received the Science Award of TUBITAK in 1994. 